\title{
Arguments that Prehistorical and Modern Humans Belong to the Same Species
}

\author{
Rainer W. Kühne \\ Tuckermannstr. 35, 38118 Braunschweig, Germany \\ e-mail: kuehne70@gmx.de
}

May 2, 2019

\begin{abstract}
I argue that the evidence of the Out-of-Africa hypothesis and the evidence of multiregional evolution of prehistorical humans can be understood if there has been interbreeding between Homo erectus, Homo neanderthalensis, and Homo sapiens at least during the preceding 700,000 years. These interbreedings require descendants who are capable of reproduction and therefore parents who belong to the same species. I suggest that a number of prehistorical humans who are at present regarded as belonging to different species belong in fact to one single species.
\end{abstract}

\section{Keywords}

Homo sapiens, Homo neanderthalensis, Homo erectus, Homo floresiensis, Neandertals, Denisovans

\section{Human Species}

During the first decades of the twentieth century, prehistorical humans were regarded as belonging not to the same genus as the modern human. According to the present nomenclature the Pithecanthropus is now known as Homo erectus erectus and the Sinanthropus as Homo erectus pekinensis. A number of humans during the last Ice Age (Cro Magnon, Aurignac, Combe Capelle) are now regarded as belonging to the same species as modern humans, Homo sapiens.

Apart from Homo sapiens and Homo neanderthalensis (classical Neandertal) there are known a number of further prehistorical human species. The African Homo helmei (known from Florisbad, South Africa), the European Homo steinheimensis (known from Steinheim, Germany), and the Asian Homo soloensis (known from the Solo river, Java) are regarded as belonging to a transition field between Homo sapiens and Homo erectus. They are sometimes called either progressive Homo erectus or archaic Homo sapiens.

A more primitive group of prehistorical humans is sometimes classified as Homo erectus, but mostly classified as belonging to different species. These include Homo antecessor, Homo cepranensis, Homo erectus, Homo ergaster, Homo georgicus, Homo heidelbergensis, Homo mauretanicus, and Homo rhodesiensis. Sometimes the more primitive Homo habilis is regarded as belonging to the same species as Homo ergaster.

A further species is Homo floresiensis, a dwarf form known from Flores, Indonesia. This species shows some anatomical characteristics which are similar to those of the more primitive humans Homo ergaster and Homo georgicus and other anatomical characteristics which are similar to those of Homo sapiens [1][2][3].

The first genetic sequences of a prehistorical human that were examined were those of Homo neanderthalensis [4]. Up to $4 \%$ of the genome of modern European and Asian humans are from Homo neanderthalensis [5][6].

Genetic examinations provide evidence of a further extinct human species. Up to $5 \%$ of the genome of modern Eastern Asian humans are from a species called Denisovans [7][8]. The Denisovan genes help modern Tibetans to cope with life at altitudes of 4,000 metres, by preventing their blood from thickening [9].

Genetic examinations provide evidence that the modern Africans had regional archaic ancestors who split from the ancestors of anatomically modern humans 700,000 years ago and interbred with modern humans 35,000 years ago [10] (see also [11][12][13]).

Evidence of interbreeding between classical Neandertals and modern Europeans is provided by a 24,500 year old fossil from Portugal which appears like a mosaic of modern sapiens and classical Neandertal anatomy [14]. Further evidence for such an interbreeding results from genetic examinations of a 40,000 year old modern 
human from Romania whose DNA shows that $6-9 \%$ of his genome are derived from a Neandertal ancestor four to six generations back [15].

Genetic examinations of a 120,000 year old bone found in Russia show that the individual had a mother who was related to a Neandertal population and a father who was related to a Denisovan population [16]. It is assumed that Neandertals and Denisovans separated from each other 430,000 years ago [17].

\section{Out-of-Africa Hypothesis}

I wish to formulate a simple version of the Outof-Africa hypothesis of human evolution. The earliest human species (Homo habilis) lived only in Africa. There it evolved to Homo ergaster. Some individuals left Africa and migrated to Eastern Europe and evolved to Homo georgicus and Homo antecessor. Later, around 600,000 years ago, other individuals left Africa and migrated to Europe and evolved to Homo heidelbergensis. Other individuals migrated to Eastern Asia and evolved to Homo erectus pekinensis and Homo erectus erectus. Around 250,000 years ago the African Homo erectus evolved to the erectus-sapiens transition field. Such fossils are known from Jebel Irhoud in Morocco [18][19] and Florisbad in South Africa (Homo helmei). Individuals of this erectus-sapiens transition field migrated until Europe where they evolved to Homo steinheimensis and Eastern Asia were they evolved to Homo soloensis. Finally, the African erectus-sapiens transition field evolved to the African Homo sapiens. Some individuals migrated to Europe and Asia where they became the modern Homo sapiens.

\section{Discussion}

This simple version of the Out-of-Africa hypothesis cannot explain why typical Neandertal characteristics (e.g. pronounced Torus supraorbitalis) appear already with Homo heidelbergensis, continue with Homo steinheimenensis, and get more pronounced with Homo neanderthalensis. This development provides evidence of a regional evolution from Homo heidelbergensis $(600,000$ years ago) to Homo neanderthalensis (40,000 years ago or less).

The genetic examinations of modern Africans provide evidence of regional evolution of archaic humans during the preceding 700,000 years until 35,000 years ago [10].

There were regional evolutions in Europe (Neandertals), Africa (archaic humans), and Asia (Denisovans) which lasted for up to 700,000 years. Afterwards there were interbreedings with modern humans. These interbreedings explain why Homo steinheimensis is a descendant of both European Homo heidelbergensis and African Homo helmei. They explain also the 24,500 year old European fossil which appears like a mosaic of Homo neanderthalensis and Homo sapiens. They explain also why Denisovan DNA is found in modern Tibetans. Finally they explain why DNA of archaic humans is found in modern Africans.

These interbreedings between Homo sapiens and archaic humans (Neandertals, Denisovans, archaic Africans), Homo heidelbergensis and Homo helmei require the existence of descendants who were capable of reproduction and parents who belonged to the same species. This is hardly possible if all of these prehistorical humans belonged to different species, as the present nomenclature requires. Therefore I suggest that most of the known prehistorical humans in fact belonged to the same species. I suggest to use the following nomenclature:

Homo sapiens sapiens
Homo sapiens neanderthalensis
Homo sapiens steinheimensis
Homo sapiens soloensis
Homo sapiens helmei
Homo sapiens mauretanicus
Homo sapiens floresiensis
Homo sapiens denisovansis
Homo sapiens rhodesiensis
Homo sapiens heidelbergensis
Homo sapiens pekinensis
Homo sapiens erectus
Homo sapiens cepranensis
Homo sapiens antecessor
Homo sapiens georgicus
Homo sapiens ergaster

It is beyond the scope of the argumentation of this paper. However, it may turn out that other, more primitive humans also interbred with the less primitive humans mentioned above. In this case it may be useful to use the nomenclature:

Homo sapiens habilis

Homo sapiens rudolfensis

Homo sapiens naledi

\section{Conclusion}

The simple version of the Out-of-Africa hypothesis of human evolution suggested in this paper is 
reasonable and quite correct. However, a complete description of human evolution must include also the regional evolutions in Europe (Neandertals), Asia (Denisovans), and Africa (archaic humans). Interbreedings between individuals who have evolved regionally and by migration from Africa have significantly contributed to the evolution of Neandertals and modern humans. Most prehistorical humans should be regarded as belonging to the same species as modern humans.

\section{References}

[1] P. Brown et al., A Small-Bodied Hominin from the Late Pleistocene of Flores, Indonesia, Nature 431 (2004) 1055-1061.

[2] M. J. Morwood et al., Archaeology and Age of a New Hominin from Flores in Eastern Indonesia, Nature 431 (2004) 1087-1091.

[3] M. Mirazon Lahr and R. Foley, Human Evolution Writ Small, Nature 431 (2004) 10431044.

[4] M. Krings et al., Neandertal DNA Sequences and the Origin of Modern Humans, Cell 90 (1997) 19-30.

[5] R. E. Green et al., A Draft Sequence of the Neandertal Genome, Science 328 (2010) 710-722.

[6] E. Callaway, Neanderthals had Outsize Effect on Human Biology, Science 523 (2015) 512-513.

[7] M. Rasmussen et al., An Aboriginal Australian Genome Reveals Separate Human Dispersals into Asia, Science 334 (2011) 9498.

[8] M. Meyer et al., A High-Coverage Genome Sequence from an Archaic Denisovan Individual, Science 338 (2012) 222-226.

[9] E. Huerta-Sanchez et al., Altitude Adaptation in Tibetans Caused by Introgression of Denisovan-Like DNA, Nature 512 (2014) 194-197.

[10] M. H. Hammer et al., Genetic Evidence for Archaic Admixture in Africa, Proceedings of the National Academy of Sciences of the United States of America 108 (2011) 1512315128.

[11] J. Lachance et al., Evolutionary History and Adaption from High-Coverage WholeGenome Sequences of Diverse African Hunter-Gatherers, Cell 150 (2012) 457-469.
[12] D. Xu et al., Archaic Hominin Introgression in Africa Contributes to Functional Salivary MUC7 Genetic Variation, Molecular Biology and Evolution 34 (2017) 2704-2715.

[13] E. M. L. Scerri et al., Did Our Species Evolve in Subdivided Populations Across Africa, and Why Does It Matter?, Trends in Ecology \& Evolution 33 (2018) 582-594.

[14] C. Duarte et al., The Early Upper Paleolithic Human Skeleton from the Abrigo do Lagar Velho (Portugal) and Modern $\mathrm{Hu}-$ man Emergence in Iberia, Proceedings of the National Academy of Sciences of the United States of America 96 (1999) 76047609 .

[15] Q. Fu et al., An Early Modern Human from Romania with a Recent Neanderthal Ancestor, Nature 524 (2015) 216-219.

[16] V. Slon et al., The Genome of the Offspring of a Neanderthal Mother and a Denisovan Father, Nature 561 (2018) 113-116.

[17] M. Meyer et al., Nuclear DNA Sequences from the Middle Pleistocene Sima de los Huesos Hominins, Nature 531 (2016) 504507.

[18] J. J. Hublin et al., New Fossils from Jebel Irhoud (Morocco) and the Pan-African Origin of Homo Sapiens, Nature 546 (2017) 289-292.

[19] D. Richter et al., The Age of Hominin fossils from Jebel Irhoud, Morocco and the Origins of the Middle Stone Age, Nature 546 (2017) 293-296. 